\title{
MARKET TIMING E AVALIAÇÃO DE DESEMPENHO DOS FUNDOS BRASILEIROS
}

RESUMO

Este trabal ho avalia o desempenho de fundos de investimento brasileiros pelo seu market timing, ou seja, pela capacidade de os gestores anteciparem diferenças de retorno das ações em relação a um ativo de renda fixa. Utilizam-se testes - paramétrico e não-paramétrico - desenvolvidos por Henriksson e Merton para a análise do desempenho de 243 fundos, no período de setembro de 1998 a outubro de 2003. Encontra-se evidência de habilidade de market timing para uma minoria de gestores de fundos, resultado que aparentemente se deve à maior facilidade de se preverem grandes diferenças de retorno entre 0 mercado acionário e a taxa de juros livre de risco.

\section{Liliana de M. C. Leusin}

Fides Asset Management

\section{Ricardo D. Brito}

Ibmec São Paulo

ABSTRACT This work evaluates the performance of Brazilian investment funds vis-à-vis their market timing, in other words, their capacity to preempt differences in the return on shares relative to a fixed income asset. Parametric and non-parametric tests (as developed by Henriksson and M erton) were used for analyzing the performance of 243 funds for the period between September 1998 and October 2003. Evidence was found of the market timing skills of a minority of fund managers, a result that is apparently due to their greater facility for forecasting any large differences in return between the share market and the risk-free interest rate.

PALAVRAS-CHAVE Market timing, seletividade, eficiência de mercado, taxa de juros, fundos de investimento.

KEYWORDS M arket timing, selection, market efficiency, interest rates, investment funds. 


\section{INTRODUÇÃO}

A indústria de fundos de investimento em ações reflete 0 dilema do investidor entre gestão passiva e ativa dos seus recursos. Enquanto a primeira estratégia simplesmente acompanha a evolução da carteira de mercado, a segunda expõe mais os recursos nos momentos de al ta esperada e protege-os quando a expectativa é de baixa. N os últimos anos, os fundos passivos têm ganhado participação em mercados competitivos, estimulados por evidências empíricas de que a gestão ativa não é capaz de gerar excessos de retorno significativos.

No Brasil, a participação dos fundos passivos no volume investido em fundos de renda variável tem se mantido baixa. Involuiu de 5,4 \% em setembro de 1998 para 0,8 \% em agosto de 2003, sinalizando um possível convencimento dos investidores da habilidade da gestão ativa de gerar retornos superiores à gestão passiva no mercado nacional.

0 objetivo deste trabal ho é avaliar o desempenho dos fundos de investimento do mercado brasileiro pelo seu market timing, ou seja, pela capacidade de antecipar movimentos no preço das ações em relação ao ativo de renda fixa. Essa análise é de interesse tanto prático como teórico. Para os investidores, permite inferir se o esforço de alocação ativa pelos gestores contribui para os retornos obtidos além da simples alocação passiva. Para os gestores, mensura a efetividade de suas estratégias ativas, podendo embasar regras de remuneração. Para os pesquisadores, trata-se de um teste de eficiência dos mercados financeiros na medida em que os grandes movimentos da taxa de juros brasileira podem facilitar a obtenção de retornos sistematicamente anormais.

Segundo Fama (1970), a habilidade de previsão pode ser separada em dois componentes, micro e macro. A previsibilidade micro, denominada sel etividade ( security sel ection), é a capacidade de previsão do movimento dos preços de ações individuais em relação às ações em geral. Em busca de sel etividade, geralmente são analisados demonstrativos de resultados de empresas, anál ises setoriais e análise gráfica comparativa entre ativos. A previsibilidade macro, denominada market timing, é a capacidade de previsão do movimento geral dos preços das ações em relação ao ativo de renda fixa. Para a obtenção de market timing, estudam-se indicadores macroeconômicos como a política monetária (principalmente a taxa de juros), a política cambial e a política tributária, dentre outros.

0 gestor quese considera capaz de prever o movimento relativo das ações ou do mercado acionário em relação à renda fixa realocará ativamente os seus recursos naquel es ativos de maior retorno esperado. Caso bem-sucedido na sel etividade, conseguirá excessos de retorno anormais e independentes do movimento do mercado. Se bem-sucedido em market timing, obterá excessos de retorno anormais que dependem do mercado de forma não linear.

A literatura internacional sobre a habilidade dos gestores em gerar retornos anormais é vasta e, em geral, conclui pela sua insignificância. Jensen (1968) avalia o desempenho de fundos americanos entre 1945 e 1964, sem fazer distinção entre sel etividade e market timing, e não encontra evidência de retornos superiores. Treynor e M azuy (1966) analisam o retorno anual de 57 fundos no mercado americano e concluem que em apenas um dos fundos a hipótese de não-habilidade de market timing é rejeitada.

Mais recentemente, Merton (1981) formaliza a análise de criação de valor por market timing, onde o gestor prevê o maior retorno do mercado em relação ao ativo sem risco, ou vice-versa, mas não consegue prever sua magnitude. Partindo dos fundamentos conceituais por ele desenvolvidos, Henriksson e Merton (1981) elaboram dois testes, um não-paramétrico e um paramétrico, para avaliar o desempenho dos fundos de acordo com o market timing. Henriksson (1984) aplica esses testes ao mercado americano para o período de fevereiro de 1968 a julho de 1980, e conclui que a habilidade em market timing é praticamente inexistente.

Brito, Bona e Tarciro (2003) aplicam as anál ises anteriores para o caso de fundos de gestão ativa com um referencial passivo (benchmark) bem definido, onde a habilidade em market timing estaria na sel eção de uma posição com apostas ativas ou uma posição no referencial passivo, de acordo com a previsão do gestor sobre qual retorno será superior. Eles propõem um índice específico para avaliar o desempenho e a eficiência em market timing dos fundos de gestão ativa. Brito (2003) analisa o segmento de fundos de derivativos no Brasil e chega à conclusão de que, numa janela de 30 dias úteis com cotações disponíveis em 31 de outubro de 1999, poucos gestores apresentaram capacidade significativa de previsão macro. Franz e Figueiredo (2003) também não identificam market timing para 29 fundos mútuos de ações brasileiros entre janeiro de 1995 e dezembro de 2000.

0 presente trabalho examina se existe evidência de habilidade em market timing na indústria de fundos brasileira de acordo com os testes desenvolvidos por Henriksson e M erton (1981). Encontra-se evidência de market timing na indústria de fundos de investimento do Brasil durante o período de setembro de 1998 a agosto de 2003, mas apenas para uma minoria dos fundos, e em momentos de grandes diferenças entre os dois mercados. 
A segunda seção deste artigo traz uma revisão bibliográfica, examinando os fundamentos conceituais e os principais model os desenvol vidos para aval iar a habilidade de market timing. Em seguida, a seção três apresenta a metodologia utilizada no trabal ho, e as hipóteses nele propostas. A quarta seção apresenta o resultado dos testes para o segmento de fundos no Brasil. A seção final sintetiza as principais conclusões do trabalho.

\section{REVISÃO BIBLIOGRÁFICA}

Merton (1981) desenvolveu um modelo para avaliar a habilidade em market timing independente da distribuição dos retornos e de um modelo de apreçamento específico; até então, sempre se havia utilizado o CAPM como modelo de apreçamento. 0 modelo de Merton (1981) é baseado no fato de que os gestores têm capacidade de prever se o retorno do mercado irá superar o retorno do ativo de renda fixa, e vice-versa, mas não têm capacidade de mensurar quanto. Assim, os gestores al ocarão seus recursos em ações ou títulos de renda fixa de acordo com a previsão de desempenho superior. A habilidade de previsão do gestor é definida no contexto de probabilidade condicional do modelo binomial em dois estágios, como ilustrado na Figura 1 . No primeiro estágio, os investidores observam a probabilidade de 0 ativo de renda fixa apresentar retorno superior às ações. No segundo, os investidores analisam a probabilidade de que os gestores prevejam corretamente qual ativo terá desempenho superior.

Pode-se representar a previsão dos gestores em t-1 pela variável binomial $\delta(\mathrm{t})$ tal que:

$$
\delta(t)=\left\{\begin{array}{ll}
=0 & \text { se } R_{f t} \geq R_{M t} \\
=1 & \text { se } R_{M t}>R_{f t}
\end{array},\right.
$$

em que $R_{f t}$ é o retorno da renda fixa e $R_{M t}$ é o retorno da carteira de mercado, com probabilidades condicionais ao retorno do mercado:

$$
P_{1 t}=P\left[\delta(t)=0 \mid R_{f t} \geq R_{M t t}\right]
$$

éa probabilidade de o gestor acertar que o retorno da renda fixa será superior ao retorno da carteira de mercado;

$$
1-P_{1 t}=P\left[\delta(t)=1 \mid R_{f t} \geq R_{M t t}\right]
$$

é a probabilidade de o gestor errar que o retorno da carteira de mercado será superior ao retorno da renda fixa;

$$
P_{2 t}=P\left[\delta(t)=1 \mid R_{M t}>R_{f t}\right]
$$

é a probabilidade de o gestor acertar que o retorno da carteira de mercado será superior ao retorno da renda fixa; e

$$
1-P_{2 t}=P\left[\delta(t)=0 \mid R_{M t}>R_{f t}\right]
$$

é a probabilidade de o gestor errar que o retorno da renda fixa será superior ao retorno da carteira de mercado.

Como $P_{1 t}$ e $P_{2 t}$ são as probabilidades de acerto nos dois estados da natureza possíveis, Merton (1981) define a

Figura 1 - A habilidade de previsão do gestor é definida em contexto de probabilidade condicional do modelo binomial em dois estágios
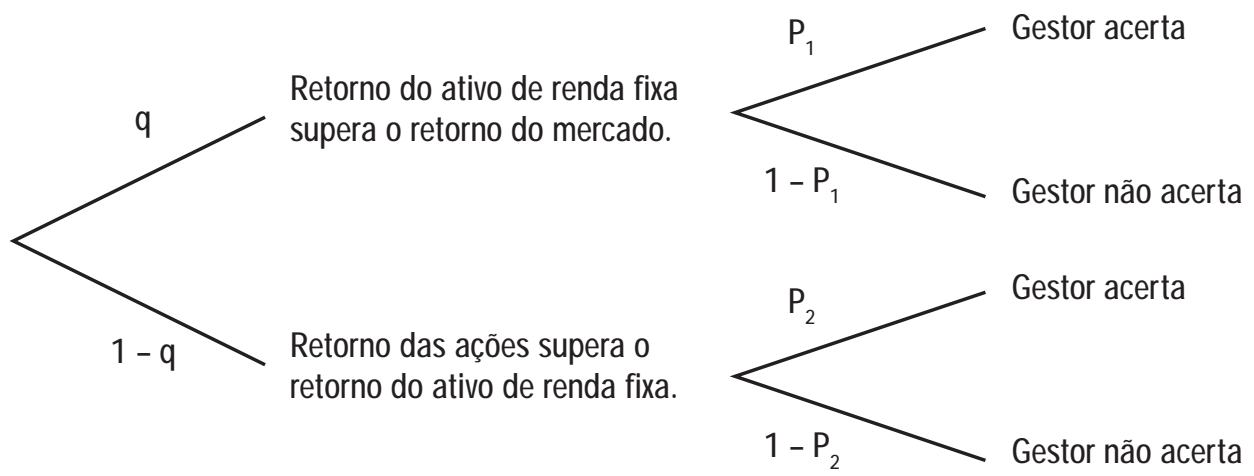
habilidade do gestor em market timing como $\left(\mathrm{P}_{1 \mathrm{t}}+\mathrm{P}_{2 \mathrm{t}}\right)$, sendo condição necessária e suficiente para que a previsão do gestor tenha valor positivo para os investidores:

$$
\left(P_{1 t}+P_{2 t}\right)>1 \text {. }
$$

Ou seja, o gestor precisa acertar mais do que errar.

É importante observar que, embora a modelagem sugira uma escolha binária do tipo renda variável ou fixa, por outros aspectos, como aversão ao risco e hedge do gestor,ou limitações regulatórias, na prática se espera uma aposta menos agressiva. Em outras palavras, alocações que possuam quantidades positivas das duas classes de ativos também podem tentar market timing, bastando que variem as proporções alocadas de acordo com as expectativas de retorno.

\section{Teste não-paramétrico para avaliação de habilidade em market timing}

Henriksson e Merton (1981) propõem examinar a habilidade de market timing a partir das probabilidades condicionais de previsão correta $\mathrm{P}_{1 \mathrm{t}}$ e $\mathrm{P}_{2 \mathrm{t}^{\prime}}$ que, por serem independentes da distribuição dos retornos, proporcionam um teste robusto.

Testa-se a hipótese nula de que não há habilidade em market timing, ou seja, $\mathrm{H}_{0}:\left(\mathrm{P}_{1 \mathrm{t}}+\mathrm{P}_{2 \mathrm{t}}\right)=1$, em que as probabilidades condicionais de uma previsão correta, $\mathrm{P}_{1 \mathrm{t}} \mathrm{e}$ $P_{2 t^{\prime}}$ não são conhecidas.

Sendo $n_{1}$ o número de previsões corretas no estado $R_{M}$ $\leq R_{f}, n_{2}$ o número de previsões erradas no estado $R_{M}>R_{f^{\prime}}$ $n=n_{1}+n_{2}$ o número de previsões em que $R_{M} \leq R_{f}, N_{1} 0$ número de observações em que $R_{M} \leq R_{f^{\prime}} N_{2}$ o número de observações em que $R_{M}>R_{f}$ e $N=N_{1}+N_{2}$ o número total de observações, recorre-se às definiç̧ões:

$$
E\left[n_{1} / N_{1}\right]=P_{1}, \quad \text { e } \quad E\left[n_{2} / N_{2}\right]=1-P_{2},
$$

e a hipótese nula $\mathrm{H}_{0}:\left(\mathrm{P}_{1 \mathrm{t}}+\mathrm{P}_{2 \mathrm{t}}\right)=1$ para escrever:

$$
E\left[n_{1} / N_{1}\right]=P_{1}=1-P_{2}=E\left[n_{2} / N_{2}\right],
$$

ou mesmo:

$$
E\left[\left(n_{1}+n_{2}\right) /\left(N_{1}+N_{2}\right)\right]=E[n / N]=P_{1}=P .
$$

Como na maioria das vezes é difícil obter as previsões dos gestores, o teste não-paramétrico é difícil de ser utilizado na prática, a não ser que se consiga uma aproximação muito boa das expectativas.

\section{Teste paramétrico para avaliação de habilidade em market timing}

Tendo em vista a dificuldade de aproximação das expectativas no teste não-paramétrico, Henriksson e Merton também apresentam um teste paramétrico para avaliar habilidade em market timing. Esse teste requer a observação dos retornos e a suposição de que os ativos são apreçados de acordo com um modelo específico. N ormalmente utiliza-se o CAPM como modelo de apreçamento, mas o teste é facilmente adaptado a outros model os que considerem múltiplos fatores, como o APT.

Jensen (1968) já havia utilizado o CAPM para avaliar o desempenho defundos entre 1945-1964, não encontrando evidência de que os gestores dos fundos possuíssem habilidade para gerar um retorno superior ao do mercado. No entanto, Jensen não separa a habilidade dos gestores entre seletividade e market timing, apenas estima a regressão:

$$
\left(R_{P t}-R_{f t}\right)=\alpha+\beta\left(R_{M t}-R_{f t}\right)+e_{P t}
$$

em que $e_{p t}$ satisfaz as condições

$$
\begin{aligned}
& E\left[e_{P t}\right]=0, E\left[e_{P t} \mid\left(R_{M t}-R_{f t}\right)\right]=0, \mathrm{e} \\
& E\left[e_{P t} \mid e_{P t-i}\right]=0 \text { para } i=1,2,3,
\end{aligned}
$$

Henriksson e M erton (1981) separam sel etividade e market timing, com foco neste último. Assumem que os gestores escolhem entre dois níveis de risco sistemático $(\beta)$ dependendo de suas previsões de retorno relativo entre a carteira de mercado e a renda fixa. Ou seja, o gestor utilizará um $\beta$ maior (mais agressivo) quando previr que $\mathrm{R}_{\mathrm{Mt}}>\mathrm{R}_{\mathrm{ft}}$ e um $\beta$ menor (mais conservador) quando previr que $R_{M t} \leq R_{f t}$, como ilustrado na Figura 2, abaixo.

Denotando $\eta_{1}$ como $0 \beta_{\mathrm{t}}$ escolhido para a carteira quando 0 gestor prevê que $\mathrm{R}_{\mathrm{Mt}} \leq \mathrm{R}_{\mathrm{ft}}$ e $\eta_{2}$ como $0 \beta_{\mathrm{t}}$ escolhido quando a previsão é de que $\mathrm{R}_{\mathrm{Mt}}>\mathrm{R}_{\mathrm{ft}}$, então $\beta_{\mathrm{t}}=\eta_{1}$ quando a previsão for de baixa no mercado e $\beta_{\mathrm{t}}=\eta_{2}$ quando a previsão for de alta no mercado. Como os agentes são racionais, então $\eta_{2}>\eta_{1}$.

Se $\beta_{\mathrm{t}}$ fosse observável em cada ponto do tempo, as previsões dos gestores seriam conhecidas e o teste nãoparamétrico poderia ser utilizado. No entanto, $\beta_{\mathrm{t}}$ é uma variável al eatória não observável. Assim, pode-se denotar b como o valor esperado de $\beta_{\mathrm{t}}$ que não depende das previsões dos gestores.

$$
b=q\left[P_{1} \eta_{1}+\left(1-P_{1}\right) \eta_{2}\right]+(1-q)\left[P_{2} \eta_{2}+\left(1-P_{2}\right) \eta_{1}\right]
$$

em que q é a probabilidade incondicional de que $R_{M t} \leq R_{\mathrm{ft}}$. 
Com uso de álgebra, chega-se à seguinte regressão a ser estimada:

$$
\begin{aligned}
& \left(R_{P t}-R_{f t}\right)=\alpha+\beta_{1}\left(R_{M t}-R_{f t}\right)+\beta_{2} \max \left[0, R_{f t}-R_{M t}\right] \\
& +e_{P t},
\end{aligned}
$$

que separa as contribuições de market timing e da seletividade para o excesso de retorno da carteira.

0 termo $\beta_{2} \max \left[0, R_{f t}-R_{M t}\right]$ mede a habilidadeem market timing uma vez que o gestor racional terá comprado em renda fixa e vendido na carteira de mercado se previr que o retorno da primeira superará o da segunda. Dessa análise, conclui-se que o retorno de um investimento com perfeita habilidade em market timing éigual ao retorno de uma estratégia que investe recursos no mercado acionário e compra opções de venda da carteira do mercado com preço de exercício $R_{\mathrm{ft}}$ (estratégia chamada de protective put ou insured equity). Como o custo de se adquirirem opções da carteira de mercado ao preço de exercício $R_{\mathrm{ft}}$ não é igual a zero, o valor total da habilidade em market timing é igual ao preço desses derivativos multiplicado pelo patrimônio do fundo.

Henriksson e M erton (1982) mostram que, para grandes amostras, a habilidade em market timing do gestor é expressa por $\hat{\beta}_{2}$. 0 verdadeiro $\beta_{2}$ é igual a zero se 0 gestor não tem habilidade de market timing $\left(\mathrm{P}_{1}+\mathrm{P}_{2}=\right.$ $1)$ ou se não age de acordo com as suas previsões $\left(\eta_{2}=\right.$ $\eta_{1}$ ). Argumentam também que 0 estimador por mínimos quadrados ordinários dos parâmetros que explicam a performance de uma carteira qual quer é consistente, porém ineficiente. Isso porque $\beta_{\mathrm{t}}$ não é estacionário. Os autores mostram ainda que essa não-estacionariedade resulta em heteroscedasticidade na medida em que o desvio padrão dos resíduos é uma função crescente de $\left|R_{M t}-R_{f t}\right|$. A correção proposta consiste em estimar a equação (2) por mínimos quadrados e utilizar o valor absoluto dos resíduos como variável dependente na regressão a seguir, reestimando a equação (2) com suas variáveis divididas por

$$
\begin{aligned}
& \left\{\hat{\phi}_{P}+\hat{\Omega}_{1 P} \min \left[0, R_{M t}-R_{f t}\right]+\hat{\Omega}_{2 P} \max \left[0, R_{M t}-R_{f t}\right]\right\} . \\
& \left|e_{P t}\right|=\phi_{P}+\Omega_{1 P} \min \left[0, R_{M t}-R_{f t}\right]+\Omega_{2 P} \max \left[0, R_{M t}-R_{f t}\right] \\
& +\xi_{P} ;
\end{aligned}
$$

\section{METODOLOGIA}

Este trabal ho aplica os testes - paramétrico e não-paramétrico - desenvolvidos por Henriksson e Merton para examinar se os gestores dos fundos de investimentos brasileiros possuem habilidade em market timing. Os dados são da base Quantum Axis, e, a partir da classificação da Anbid, foram selecionadas as categorias de fundos: ações

Figura 2 - Os gestores escolhem entre diferentes níveis de risco sistemático dependendo da sua previsão

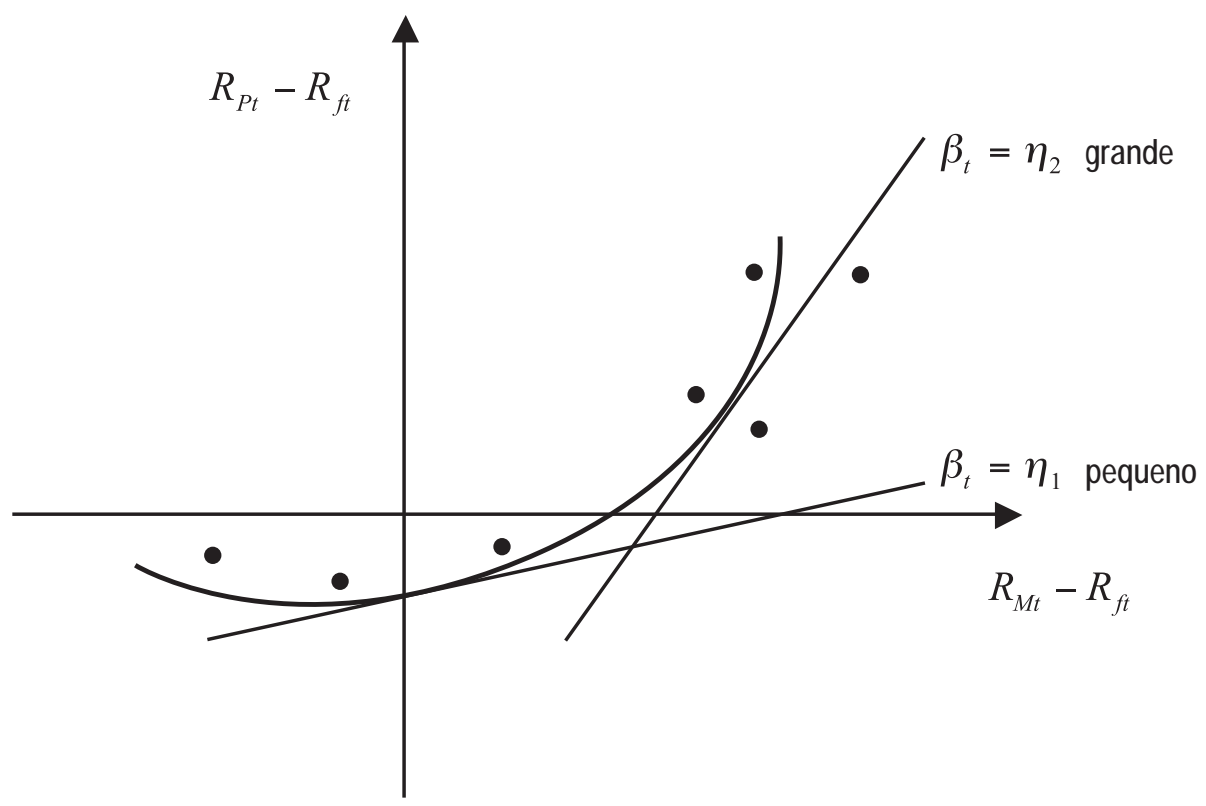


Ibovespa ativo, ações Ibovespa ativo com alavancagem, ações IBX ativo, ações IBX ativo com alavancagem, outros fundos de ações, fundos multimercado sem al avancagem com renda variável, fundos multimercado com al avancagem com renda variável e fundos multimercado balanceados Se para os primeiros o referencial é claro, para os últimos não é formalmente explicitado. Para fins deste estudo, mais importante que o referencial é que sejam fundos cujas composições das carteiras permitam a utilização de renda variável e fixa com percentual à escolha do gestor, ou seja, gestão ativa. A listagem dos fundos da amostra, inclusive suas classificações na Anbid, en contrase no Quadro 1.

Os retornos mensais são computados em taxas contínuas,

Quadro 1 - Amostra dos fundos de investimentos

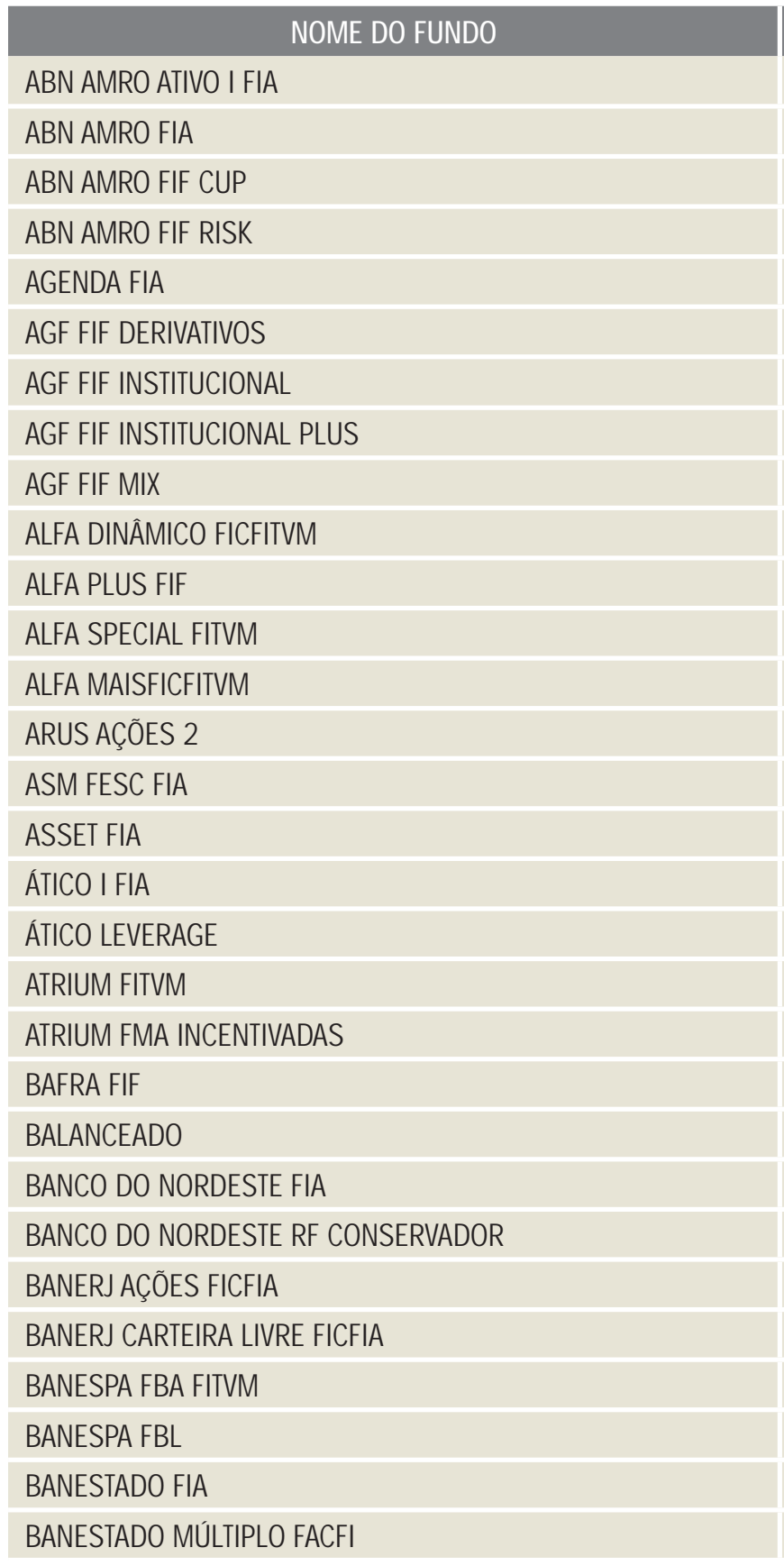

\section{CALSSIFICAÇÃO ANBID}

AÇÕES IBOVESPAATIVO

AÇÕES IBXATIVO

MULTTMERCADOCOM RV

MULTIMERCADOCOM RVCOMALAVANCAGEM

AÇÕES IBOVESPAATIVO

MULTIMERCADOCOMRV

MULTMERCADOCOMRV

MULTIMERCADOCOMRV

MULTMERCADOCOM RV

AÇÕES IBOVESPAATIVO

MULTMERCADOCOM RV

AÇÕES IBOVESPAATIVO

AÇÕES IBOVESPAATIVO

AÇÕES IBOVESPAATIVO

AÇÕES IBXATIVO

AÇÕES OURROS

AÇÕES IBOVESPAATIVO

MULTMERCADOCOM RVCOMALAVANCAGEM

AÇÕES OUIROS

AÇÕES OUIROS

MULTIMERCADOCOM RVCOMALAVANCAGEM

BALANCEADOS

AÇÕES IBOVESPAATIVO

BALANCEADOS

AÇÕES IBXATIVO

MULTIMERCADOCOM RVCOMALAVANCAGEM

AÇÕES OURROS

AÇÕES OUIROS

AÇÕES IBXATIVO

MULTIMERCADOCOM RVCOMALAVANCAGEM

(continua) 


\begin{tabular}{|c|c|}
\hline BANESTESAÇÕES & AÇÕES OURROS \\
\hline BANIF PRIMUS AÇÕES & AÇÕES IBXATIVO \\
\hline BANIF PRIMUS DERVATIIOS & MULTMERCADOCOM RVCOMALAVANCAGEM \\
\hline BANRISULÍNDICE & AÇÕES IBOVESPAATIVO \\
\hline BANRSULINFRA & AÇÕES OURROS \\
\hline BANRISULPERFORMANCE FIMM & AÇÕES IBXATIVO \\
\hline BBAÇÕES MASTER & AÇÕES IBOVESPAATIVO \\
\hline BBAAQARIUS & BALANCEADOS \\
\hline BBAFF LEADER & MULTMERCADOCOMRV \\
\hline BBAFF SPEQAL & MULTMERCADOCOM RVCOMALAVANCAGEM \\
\hline BBAIBOVESPAINSTITUCONAL FA & AÇÕES IBOVESPAATIVO \\
\hline BBA IBOVESPA PRIVATE FA & AÇÕES IBOVESPAATIVO \\
\hline BBAIBXINSTITUCONALFA & AÇÕES IBXATIVO \\
\hline BBMAÇÕES & AÇÕES IBXATIVO \\
\hline BONACTIVE FA & AÇÕES IBOVESPAATIVOCOMALAVANCACEM \\
\hline BON FABLE OHP & AÇÕES IBOVESPAATIVO \\
\hline BCNFASEGURIDADE & AÇÕES IBOVESPAATIVO \\
\hline BON MAIS FF & MULTMERCADOCOM RVCOMALAVANCAGEM \\
\hline BCN PERFORMANCE & MULTMERCADOCOM RVCOMALAVANCAGEM \\
\hline BCN SUPERAÇÃOFA & AÇÕES IBOVESPAATIVO \\
\hline BEMGEAÇÕES HCFA & AÇÕES IBXATIVO \\
\hline BESC PBA & AÇÕES OUIROS \\
\hline BESCMXFQ & AÇÕES OUTROS \\
\hline BMGFIA & AÇÕES IBOVESPAATIVO \\
\hline BNP PARIBAS EQUTY & AÇÕES IBOVESPAATIVO \\
\hline BNP PARIBAS PREVER C & MULTIMERCADOCOM RV \\
\hline BNP PARIBAS TARGETHF & MULTIMERCADOCOMRV \\
\hline BOSTON IBOVESPASEECTRA & AÇÕES IBOVESPAATIVO \\
\hline BOSTOPORTFOUO & MULTMERCADOCOM RVCOMALAVANCAGEM \\
\hline BOSTON PRIVATE IBOVESPAPLUS & AÇÕES IBOVESPAATIVO \\
\hline BOSTONSMAL CAPS VALUATION & AÇÕES OUIROS \\
\hline BOSTON STRATEGY & MULTMERCADOCOM RVCOMALAVANCAGEM \\
\hline BRADESCOBDFA & AÇÕES IBOVESPAATIVOCOMALAVANCAGEM \\
\hline BRADESCOBJ FIACASSIC & AÇÕES IBOVESPAATIVOCOMALAVANCAGEM \\
\hline BRADESCOBJ FIAENERGA & AÇÕES IBOVESPAATIVOCOMALAVANCAGEM \\
\hline BRADESCOBJ FIAEQUTIES & AÇÕES IBOVESPAATIVO \\
\hline BRADESCOBJ FIAIMESTORS & AÇÕES IBXATIVO \\
\hline BRADESCOBJ FIANUMBERONE & AÇÕES IBOVESPAATIVOCOMALAVANCAGEM \\
\hline
\end{tabular}


(continua)

BRADESCOBJ FIF STRATEGC TRADER BRADESCO FAPREMUM

BRADESCO GODEN PROATCONSERVADOR

BRADESCO GODEN PROFTDINÂMICO

BRADESCO GODEN PROFTMODERADO

BRADESCO II FA

BRADESCOUVREFÁCL

BRB FTMM

CCF BANKNNG FA

COF GESTÃO

CCF GOD

COF INSTITUCONALFA

CCF UBRUUM

QTT INSTITUAONALAÇÕES FA

वTा AÇÕES FA

QTI AÇÕES RENDAMISTAFA

CTIBRAZLBOND

ATIPUNDOS FOOUS FCFIA

COMNALORES AÇÕES HQFA

COINALORES DIAMOND

COINALORES FATOR FA

COMNALORES HSBCAÇÕES HQFA

COMNALORES REGA

COMERAALAF

COMEROAL MASTERFA

CONFANÇAE FF

CONFANÇAF FF

CONFANÇA GFF

CSAMARRODITE

CSAMFGPREMUMFIA

CSAMHGHMEDI

DYNAMOCOUGAR

EITEA

ENNESAFA

FAC CARIERA PRIVATE 102C

FAMACHAUENGER FA

FATOR PLURALEXIRAFF

FATOR PLURALFIAUVE
MULTIMERCADOCOM RVCOMALAVANCAGEM

AÇÕES IBOVESPAATIVO

BALANCEADOS

BALANCEADOS

BALANCEADOS

AÇÕES IBOVESPAATIVO

AÇÕES OUTROS

AÇÕES IBOVESPAATIVOCOMALAVANCAGEM

AÇÕES OUTROS

MULTIMERCADOCOM RV

AÇÕES IBXATIVO

AÇÕES IBXATIVO

AÇÕES OUIROS

AÇÕES IBOVESPAATIVO

AÇÕES IBOVESPAATIVO

BALANCEADOS

ULTIMERCADOCOMRV

AÇÕES IBOVESPAATIVO

AÇÕES IBXATIVO

MULTIMERCADOCOM RVCOMALAVANCAGEM

AÇÕES IBOVESPAATIVOCOMALAVANCACEM

AÇÕES IBXATIVO

MULTIMERCADOCOM RVCOMALAVANCAGEM

MULTMERCADOCOM RV

ACOÕES IBOVESPAATIVOCOMALAVANCACEM

MULTIMERCADOCOM RV

MULTIMERCADOCOM RVCOMALAVANCAGEM

MULTMERCADOCOMRV

AÇÕES IBXATIVO

AÇÕES IBOVESPAATIVOCOMALAVANCACEM

MULTIMERCADOCOM RVCOMALAVANCAGEM

AÇÕES OUTROS

AÇÕES IBOVESPAATIVO

AÇÕES OUTROS

MULTIMERCADOCOM RVCOMALAVANCAGEM

AÇÕES IBOVESPAATIVO

MULTIMERCADOCOM RVCOMALAVANCAGEM

AÇÕES IBOVESPAATIVO 


\begin{tabular}{|c|c|}
\hline FATORPLURAL HEDGE HF & MULTMERCADOCOM RVCOMALAVANCAGEM \\
\hline FATOR PLURAL INSTITUCONAL FA & AÇÕES IBOVESPAATIVO \\
\hline FATOR PLURALJAGAAR & ACCÕES IBOVESPAATIVOCOMALAVANCAGEM \\
\hline F̂́NIXFF & MULTMERCADOCOMRV \\
\hline FAANTARES & AÇÕES IBXATIVO \\
\hline FASIGMA & AÇÕES IBOVESPAATIVOCOMALAVANCAGEM \\
\hline FBRAFCFF & BALANCEADOS \\
\hline FBRA PERFORMANCE FF & MULTMERCADOCOM RVCOMALAVANCAGEM \\
\hline FBRAИCFA & AÇÕES IBOVESPAATIVO \\
\hline FDEIDADEL FF & MULTMERCADOCOMRV \\
\hline FF PILAR & MULTMERCADOCOM RV \\
\hline FNASAAÇÕES III & AÇÕES IBOVESPAATIVO \\
\hline RNASAAÇÕES ÍNDICE & AÇÕES IBOVESPAATIVO \\
\hline FOREGNPUND & MULTMERCADOCOM RV \\
\hline GAP EDCE FF & MULTMERCADOCOM RVCOMALAVANCAGEM \\
\hline GAPIBOVESPAPLUS HA & AÇÕES IBOVESPAATIVOCOMALAVANCAGEM \\
\hline GERACÃOFA & AÇÕES OURROS \\
\hline GUARARAPES FTM & AÇÕES IBOVESPAATIVO \\
\hline HEDGNG-GNFOTOP FFF & MULTMERCADOCOM RVCOMALAVANCAGEM \\
\hline HEDGNG-GNFOVERDE HF & MULTMERCADOCOM RVCOMALAVANCAGEM \\
\hline HGTOPAÇÕES & AÇÕES OUIROS \\
\hline HSBCAÇÕES HTM & AÇÕES IBOVESPAATIVOCOMALAVANCACEM \\
\hline HSBCACUMULAÇÃOFA & AÇÕES IBXATIVO \\
\hline HSBCBLUEOHP FTM & AÇÕES IBOVESPAATIVO \\
\hline HSBCDINÂMCOBAFIF & MULTMERCADOCOM RVCOMALAVANCAGEM \\
\hline
\end{tabular}

líquidos das taxas de administração e performance. A escolha da freqüência mensal e não diária, al ém de acordar com Henriksson (1984), sejustifica pela menor curtose dos retornos da primeira e pelo fato de as flutuações da taxa de juros terem freqüência mais baixa que a segunda. A subtração das taxas visa tornar os retornos comparáveis para a escol ha do investidor, que perceberá val or na habilidade de market timing apenas se auferir excesso de retorno líquido sobre a estratégia passiva. São analisados os retornos mensais de 243 fundos de investimento de setembro de 1998 a agosto de 2003, que representavam, respectivamente, $50 \%$ e $74 \%$ do mercado brasileiro de fundos com investimentos em ações. Utiliza-se o I bovespa como carteira de mercado e o swap pré-30 dias como ativo de renda fixa.

Para realizar o teste paramétrico, utiliza-se o CAPM como modelo de apreçamento, assim como a maioria (condusão)

AVANCAGEM

ACÕES IBOVESPAATIVOCOMALAVANCAGEM

MULTMERCADOCOM RV

AÇÕES IBXATIVO

AÇÕES IBOVESPAATIVOCOMALAVANCAGEM

DANCEDOS

MULTIMERCADOCOM RVCOMALAVANCAGEM

AÇOEES IBOVESPAATIVO

MULTTMERCADOCOM RV

AÇÕES IBOVESPAATIVO

AÇÕES IBOVESPAATIVO

MULTMERCADOCOM RV

AÇÕES IBOVESPAATIIOCOMALAVANCAGEM

AÇÕES OUIROS

AÇÕES IBOVESPAATIVO

MULTIMERCADOCOM RVCOMALAVANCAGEM

MULTMERCADOCOM RVCOMALAVANCAGEM

ACCÕES OUTROS

AÇÕES IBOVESPAATIVOCOMALAVANCAGEM

AÇÕES IBXATIVO

MULTMERCADOCOM RVCOMALAVANCAGEM

dos estudos realizados sobre o tema. A regressão (2), que possibilita separar as contribuições das previsões micro (seletividade) e macro (market timing), é estimada por mínimos quadrados ordinários. Em seguida, comparamse os resultados obtidos com os resultados da regressão (1), que ignora o market timing, e com a regressão (2), reestimada com correção para heterocedasticidade.

Testa-se também se os gestores apresentam habilidade para prever grandes movimentos de mercado. Para tanto, divide-se a amostra em duas pela magnitude do excesso de retorno do mercado. Os períodos em que o valor absoluto do excesso de retorno do mercado é maior que a mediana da série são separados dos períodos em que ocorre o inverso.

Por fim, realiza-se um teste não-paramétrico robusto para a especificação do modelo de apreçamento. Estima- 
se $\mathrm{P}_{1}$ e $\mathrm{P}_{2}$ a partir dos retornos do fundo e da carteira de mercado, contando quantas vezes o primeiro supera o segundo, condicionado à $R_{M t}>R_{f t}$ ou à $R_{M t} \leq R_{f t}$. Utilizando as estimativas de $P 1$ e $P 2$, testa-se a hipótese nula de não-previsibilidade, $H_{0}:\left(P_{1}+P_{2}\right)=1$.

\section{RESULTADOS}

N esta seção, são apresentados os resultados dos testes paramétrico e não-paramétrico respectivamente.

\section{Teste paramétrico}

As estimativas de mínimos quadrados ordinários da equação (2) são apresentadas na Tabela 1.0 coeficiente médio de $\beta_{2}$ positivo e significante indica market timing para 0 conjunto dos gestores. A pesar de o market timing médio ser significante, constata-se que uma minoria de gestores possui essa habilidade, com apenas 27 fundos (11,11\% da amostra) apresentando $\beta_{2}>0$ ao nível de $5 \%$ de significância. A assimetria positiva dos $\beta_{2}$ s indica al guma racionalidade na escolha de uma postura ativa.

Ainda na Tabela 1, percebe-se seletividade média negativa e significante, apesar de baixa. Sujeita à correta especificação do modelo, essa evidência indica que os esforços de sel eção entre ativos (security selection) pelo conjunto de gestores não foram bem sucedidos. Praticamente, não há evidência de habilidadeindividual deseletividade, pois apenas quatro fundos ( $1,65 \%$ da amostra) apresentam $\alpha$ $>0$ com $5 \%$ de significância na equação (2).

A Tabela 2 reporta as estimativas da regressão (1), em que a habilidade de market timing não é separada da seletividade. Os alfas estimados são superiores aos da Tabela 1 , o que se explica pela omissão do $\beta_{2}$, positivo em média. $\mathrm{N}$ esse caso, 0 alfa médio positivo e significante indica que a estratégia - combinando market timing e seleção - do conjunto dos gestores foi capaz de gerar retornos levemente superiores aos da estratégia passiva. De acordo

Tabela 1 - Teste paramétrico: $R_{P t}-R_{f t}=\alpha+\beta_{1}\left(R_{M t}-R_{f t}\right)+\beta_{2} \max \left[0, R_{f t}-R_{M t}\right]+e_{P t i} ; 243$ fundos; entre setembro de 1998 e agosto de 2003

\begin{tabular}{|c|c|c|}
\hline & MÉDIA & DESVIO PADRÃO \\
\hline alpha & $-0,003^{*}$ & 0,0009 \\
\hline beta 1 & $0,659 *$ & 0,0319 \\
\hline beta 2 & $0,133^{*}$ & 0,0294 \\
\hline \multirow[t]{2}{*}{$r^{2}$} & 0,622 & \\
\hline & \# DE FUNDOS & $\%$ AMOSTRA \\
\hline alpha (+) a 1\%de significância & 0 & $0,00 \%$ \\
\hline alpha (†) a 5\% de significância & 4 & $1,65 \%$ \\
\hline alpha (+) a 10\% de significância & 10 & $4,12 \%$ \\
\hline alpha (-) a 1\%de significância & 6 & $2,47 \%$ \\
\hline alpha (-) a 5\%de significância & 16 & $6,58 \%$ \\
\hline alpha (-) a 10\% de significância & 19 & $7,82 \%$ \\
\hline beta 2 (†) a 1\% de significância & 15 & $6,17 \%$ \\
\hline beta 2 (+) a 5\% de significância & 27 & $11,11 \%$ \\
\hline beta $2(+)$ a 10\% de significância & 51 & $20,99 \%$ \\
\hline beta 2 (-) a 1\%de significância & 1 & $0,41 \%$ \\
\hline beta 2 (-) a 5\% de significância & 4 & $1,65 \%$ \\
\hline beta 2 (-) a 10\%de significância & 6 & $2,47 \%$ \\
\hline alpha $>0$ & 84 & $34,57 \%$ \\
\hline beta $2>0$ & 175 & $72,02 \%$ \\
\hline
\end{tabular}

* indica rejeição da hipótese de coeficiente nulo ao nível de significância de 1\%; (+) denota o número de fundos com estimativas significativamente positivas; (-) denota o número de fundos com estimativas significativamente negativas. 
com os resultados da Tabela 1, uma minoria de gestores demonstra habilidade individual, com apenas 15 fundos (6,17\% da amostra) apresentando $\alpha>0$, ao nível de significância de $5 \%$. Há alguma coincidência de diagnósticos entre as estimativas das Tabelas 1 e 2. Por exemplo, com $10 \%$ de significância, dos 27 fundos com $\alpha>0,10$ fundos também apresentam estimativas de $\beta_{2}>0$, e 1 fundo apresenta $\alpha>0$ na equação (2) da Tabela 1.

Para descartar a suspeita de que os resultados da Tabela 1 são sensíveis a heterocedasticidade, a Tabela 3 apresenta as estimativas da regressão (2) corrigida segundo a equação (3). Não se percebe mudança qualitativa da evidência média de market timing positivo e seletividade negativa, nem da posse desses atributos por uma minoria dos gestores. A pequena diferença é o maior número de alfas significantes, o que não chega a indicar capacidade de seletividade do conjunto dos gestores, pois a quantidade de fundos com al fas significantemente positivos não é maior que a de negativos.

Os testes de market timing dependem da hipótese de que as probabilidades condicionais de previsões corretas sejam uniformes para todo o interval o analisado, não importando a magnitude do excesso de retorno do mercado. No entanto, a habilidade em market timing não deve ser uniforme, pois os gestores devem prever mais facilmente grandes diferenças entre a carteira de mercado e a renda fixa do que diferenças pequenas.

Para testar essa suspeita, divide-se a amostra em duas, de acordo com a magnitude do valor absoluto do excesso de retorno do mercado. Os meses com $\left|R_{M t}-R_{f t}\right|$ maior do que a mediana da série são separados dos meses em que esse valor é menor que a mediana. Para o período analisado, a mediana do valor absoluto do excesso de retorno do mercado é 7,00\% ao mês.

A Tabela 4 resume os resultados da análise das duas subamostras. De acordo com a intuição, observa-se market timing médio positivo e significante para os grandes movimentos e insignificante para os pequenos. Da análise individual, confirma-se que essa habilidade dota uma minoria de gestores.

Surpreendentemente, nenhum fundo com market timing de pequenos movimentos de mercado apresenta simultaneamente market timing de grandes movimentos. Esse resultado não é intuitivo, na medida em que gestores com sensibilidade para prever pequenos movimentos do mercado deveriam antecipar também grandes movimentos. No entanto, é um resultado possível, uma vez que o número de fundos com market timing significantemente positivo em pequenos movimentos de mercado é reduzido (apenas 2,47\% da amostra, ao nível de significância de $10 \%$ ), não se podendo descartar que o tenham conseguido por sorte.

Henriksson (1984) realizou estudo semelhante no mercado norte-americano, no período de fevereiro de 1968 a julho de 1980, e não encontrou evidência da capacidade dos gestores de preverem maiores movimentos do mercado superior a sua capacidade de preverem movimentos pequenos. Como os juros americanos flutuaram num intervalo menor que os brasileiros, os resultados de Henriksson talvez se expliquem pelo fato de os mo-

Tabela 2 - Teste paramétrico: $R_{P t}-R_{f t}=\alpha+\beta_{1}\left(R_{M t}-R_{f t}\right)+e_{P i} ; 43$ fundos; entre setembro de 1998 e agosto de 2003

\begin{tabular}{l|c|c|}
\hline & MÉDIA & DESVIO PADRÃO \\
\hline alpha & $0,003^{*}$ & 0,0006 \\
beta & $0,595^{*}$ & 0,0263 \\
\hline R2 & 0,612 & \\
\hline & \# DE FUNDOS & \% AM OSTRA \\
\hline alpha (+) a 1\%de significância & 10 & $4,12 \%$ \\
\hline alpha (+) a 5\%de significância & 15 & $6,17 \%$ \\
alpha (+) a 10\% de significância & 27 & $11,11 \%$ \\
\hline alpha (-) a 1\%de significância & 10 & $4,12 \%$ \\
\hline alpha (-) a 5\%de significância & 12 & $4,94 \%$ \\
alpha (-) a 10\% de significância & 16 & $6,58 \%$ \\
\hline alpha $>0$ & 164 & $67,49 \%$
\end{tabular}

* indica rejeição da hipótese de coeficiente nulo ao nível de significância de 1\%; (+) denota o número de fundos com estimativas significativamente positivas; (-) denota o número de fundos com estimativas significativamente negativas. 
vimentos do mercado norte-americano de capitais não serem suficientemente amplos e persistentes.

Examinando melhor os dados, percebe-se que há correlação negativa entre $\alpha$ e $\beta_{2}$. Conforme se observa na Tabela 1, 51 de 84 fundos com $\alpha$ positivo apresentam $\beta_{2}$ negativo, e 141 dos 158 fundos com $\alpha$ negativo apresentam $\beta_{2}$ positivo. Nenhum fundo apresenta ambos - market timing e seletividade - positivos ao nível de $10 \%$ de significância.

A correlação entre $\alpha$ e $\beta_{2}$ implica que fundos que apresentam retorno superior devido à seletividade possuem market timing negativo, comprometendo seu desempenho. Tal constatação contraria a análise desenvolvida por Treynor e Black (1973), que mostra que os gestores podem separar suas atividades de seleção de ativos da decisão do nível risco de mercado ao qual o fundo estará exposto (market timing).

A correlação negativa entre $\alpha$ e $\beta_{2}$ pode ser parcialmente explicada pel o fato de o I bovespa não replicar exatamente o mercado, na medida em que não contém todos as ações da economia na proporção de mercado que representam. N o entanto, as correl ações são tão fortes que enfraquecem essa explicação para o fato.

\section{Teste não-paramétrico}

A correlação negativa entre $\alpha$ e $\beta_{2}$ fomenta o questionamento à especificação do model o utilizado, cuja validade é suposta no teste paramétrico. 0 teste não-paramétrico é robusto à especificação incorreta ou à omissão de fatores relevantes, e, por isso, trata-se de procedimento indicado para esta situação.

Apesar de não requerer um model o específico de retorno, o teste requer que a previsão do gestor seja conhecida ou que se tenha uma estimativa dela. Como a previsão do gestor não é conhecida, e as estimativas podem enviesar o procedimento, opta-se pelo retorno efetivo do fundo

Tabela 3 - Teste paramétrico: $R_{P_{t}}-R_{f t}=\alpha+\beta_{1}\left(R_{M t}-R_{f t}\right)+\beta_{2} \max \left[0, R_{f t}-R_{M t}\right]+e_{P t}$ i com correção de heterocedasticidade; 243 fundos; entre setembro de 1998 e agosto de 2003

\begin{tabular}{|c|c|c|}
\hline & MÉDIA & DESVIO PADRÃO \\
\hline alpha & $-0,002^{*}$ & 0,0005 \\
\hline beta 1 & $0,630^{*}$ & 0,0294 \\
\hline beta 2 & $0,091^{*}$ & 0,0170 \\
\hline \multirow[t]{2}{*}{$\mathrm{R} 2$} & 0,631 & \\
\hline & \# DE FUNDOS & $\%$ AMOSTRA \\
\hline alpha (+) a 1\%de significância & 10 & $4,12 \%$ \\
\hline alpha (+) a 5\% de significância & 13 & $5,35 \%$ \\
\hline alpha (+) a 10\% de significância & 23 & $9,47 \%$ \\
\hline alpha (-) a 1\% de significância & 15 & $6,17 \%$ \\
\hline alpha (-) a 5\% de significância & 22 & $9,05 \%$ \\
\hline alpha (-) a 10\% de significância & 27 & $11,11 \%$ \\
\hline beta $2(+)$ a 1\% de significância & 15 & $6,17 \%$ \\
\hline beta 2 (†) a 5\% de significância & 32 & $13,17 \%$ \\
\hline beta $2(+)$ a 10\% de significância & 41 & $16,87 \%$ \\
\hline beta 2 (-) a 1\%de significância & 0 & $0,00 \%$ \\
\hline beta 2 (-) a 5\% de significância & 6 & $2,47 \%$ \\
\hline beta 2 (-) a 10\% de significância & 11 & $4,53 \%$ \\
\hline alpha $>0$ & 98 & $40,33 \%$ \\
\hline beta $2>0$ & 162 & $66.67 \%$ \\
\hline
\end{tabular}

* indica rejeição da hipótese de coeficiente nulo ao nível de significância de 1\%; (+) denota o número de fundos com estimativas significativamente positivas; (-) denota o número de fundos com estimativas significativamente negativas. 
como medida de performance, comparando-o com o desempenho do Ibovespa, condicionado ao retorno deste último em relação à renda fixa. 0 teste analisa a performance total do fundo por meio da soma das probabilidades $\left(P_{1}+P_{2}\right)$, fornecendo uma medida de valor da estratégia ativa do fundo em relação à estratégia passiva. Se o gestor acerta mais do que erra, a soma das probabilidades é maior do que um.

Os resultados do teste não-paramétrico estão resumidos na Tabela 5. A estimativa da soma das probabilidades, de 1,044 , indica que, apesar de pequena, é significantemente maior que um, indicando superioridade de desempenho da média das estratégias dos fundos ativos sobre a estratégia passiva.

Da análise individual dos fundos, confirma-se que uma minoria de gestores tem capacidade preditiva significante.
Apenas 16 fundos de investimento (6,58\% da amostra) apresentam soma de probabilidades superior a um com $5 \%$ de significância. Percebe-se também al guma coincidência de resultados entre os testes paramétrico e não-paramétrico. Ao nível de $10 \%$ de significância, dos 27 fundos com soma de probabilidades maior que um , 7 também apresentam estimativas de $\beta_{2}>0$, e 4 s possuem $\alpha>0$ na equação (2) da Tabela 1. Relativamente à equação (1) da Tabela 2, 10 dos 27 fundos com soma de probabilidades também apresentam estimativas de $\alpha>0$.

Finalmente, utiliza-se o teste não-paramétrico para verificar se os gestores têm mais facilidade de prever grandes diferenças entre a carteira de mercado e a renda fixa. Novamente, divide-se a amostra em duas, segundo a mediana de $\left|R_{M t}-R_{f t}\right|$, e procede-se ao teste das duas subamostras.

Tabela 4 - Teste paramétrico: $R_{P t}-R_{f t}=\alpha+\beta_{1}\left(R_{M t}-R_{f t}\right)+\beta_{2} \max \left[0, R_{f t}-R_{M t}\right]+e_{P t} ;$ amostra dividida pela magnitude de $\left|R_{M t}-R_{f t}\right| ; 243$ fundos; entre setembro de 1998 e agosto de 2003

\begin{tabular}{|c|c|c|c|c|}
\hline & \multicolumn{2}{|c|}{ PEQUENAS MAGNITUDES } & \multicolumn{2}{|c|}{ GRANDES MAGNITUDES } \\
\hline & MÉDIA & DESVIO PADRÃO & MÉDIA & DESVIO PADRÃO \\
\hline alpha & $-0,000$ & 0,0008 & $-0,003^{*}$ & 0,0012 \\
\hline beta 1 & $0,513^{*}$ & 0,0330 & $0,644^{*}$ & 0,0283 \\
\hline beta 2 & 0,010 & 0,0388 & $0,106 *$ & 0,0203 \\
\hline \multirow[t]{2}{*}{$\mathrm{R} 2$} & 0,436 & & 0,675 & \\
\hline & \# DE FUNDOS & $\%$ AMOSTRA & & \\
\hline alpha (+) a 1\%de significância & 6 & $2,47 \%$ & 0 & $0,00 \%$ \\
\hline alpha (+) a 5\%de significância & 15 & $6,17 \%$ & 1 & $0,41 \%$ \\
\hline alpha (+) a 10\% de significância & 24 & $9,88 \%$ & 4 & $1,65 \%$ \\
\hline alpha (-) a 1\%de significândia & 1 & $0,41 \%$ & 7 & $2,88 \%$ \\
\hline alpha (-) a 5\% de significância & 3 & $1,23 \%$ & 13 & $5,35 \%$ \\
\hline alpha (-) a 10\% de significância & 7 & $2,88 \%$ & 22 & $9,05 \%$ \\
\hline beta 2 (+) a 1\% de significância & 1 & $0,41 \%$ & 15 & $6,17 \%$ \\
\hline beta 2 (+) a 5\% de significância & 2 & $0,82 \%$ & 20 & $8,23 \%$ \\
\hline beta 2 (+) a 10\% de significância & 6 & $2,47 \%$ & 25 & $10,29 \%$ \\
\hline beta 2 (-) a 1\%de significância & 3 & $1,23 \%$ & 1 & $0,00 \%$ \\
\hline beta 2 (-) a 5\% de significância & 14 & $5,76 \%$ & 1 & $0,00 \%$ \\
\hline beta 2 (-) a 10\% de significândia & 26 & $10,70 \%$ & 3 & $0,00 \%$ \\
\hline alpha $>0$ & 146 & $60,08 \%$ & 97 & $39,92 \%$ \\
\hline beta $2>0$ & 89 & $36,63 \%$ & 168 & $69,14 \%$ \\
\hline
\end{tabular}

* indica rejeição da hipótese de coeficiente nulo ao nível de significância de 1\%; (+) denota o número de fundos com estimativas significativamente positivas; (-) denota o número de fundos com estimativas significativamente negativas, 
A Tabela 6 confirma os resultados da Tabela 4, com uma estimativa de 1,106 para a soma das probabilidades, significantemente maior que um para grandes movimentos, e de igual a 0,972 para pequenos movimentos.

Individualmente, uma pequena proporção dos fundos apresenta capacidade preditiva significante para grandes diferenças (36 fundos ao nível de 5\% de significância), mas praticamente não se verifica essa habilidade para pequenas diferenças (nenhum fundo ao nível $5 \%$ de significância).

\section{CONCLUSÕES}

Diferentemente de outros estudos sobre o tema, encontrase evidência fraca de habilidade em market timing para o mercado brasileiro de fundos de investimento de setem- bro de 1998 a agosto de 2003, primordialmente decorrente da maior capacidade de alguns gestores em prever grandes diferenças de retorno entre a carteira de mercado e a renda fixa.

Da comparação com o clássico trabal ho de Henriksson (1984), que não encontra market timing significativo no mercado americano, infere-se que os gestores antecipam e exploram melhor os movimentos nos preços das ações em relação ao ativo de renda fixa num ambiente mais instável,com taxas de juros mais variáveis, como o Brasil.

A se repetir o padrão do período analisado, tal evidência sugere ser factível e mais eficiente uma estratégia de gestão passiva condicional à relativa estabilidade, mas que explore ativamente os momentos de maior turbulência.

Ao se tomar essa situação como um fato estilizado, motiva-se o desenvolvimento de uma explicação teórica,

Gabela 5 - Teste não-paramétrico: retorno do fundo versus retorno da estratégia passiva, $H_{o}:\left(P_{1}+P_{2}\right) \leq 1 ; 243$ fundos; entre setembro de 1998 e agosto de 2003

\begin{tabular}{|l|c|c|}
\hline & MÉDIA & DESVIO PADRÃO \\
\hline$\left(\mathrm{P}_{1}+\mathrm{P}_{2}\right)$ & $1,044^{*}$ & 0,102 \\
& \# DE FUNDOS & \% AM 0STRA \\
\hline $\mathrm{P}_{1}+\mathrm{P}_{2}>1$ a $1 \%$ de significância & 12 & $4,94 \%$ \\
$\mathrm{P}_{1}+\mathrm{P}_{2}>1$ a $5 \%$ de significância & 16 & $6,58 \%$ \\
$\mathrm{P}_{1}+\mathrm{P}_{2}>1$ a $10 \%$ de significância & 27 & $11,11 \%$ \\
\hline $\mathrm{P}_{1}+\mathrm{P}_{2}>1$ & 169 & $69,55 \%$ \\
\hline
\end{tabular}

* indica rejeição da hipótese $H_{o}:\left(P_{1}+P_{2}\right) \leq 1$ ao nível de significância de $1 \%$.

Tabela 6 - Teste não-paramétrico: retorno do fundo versus retorno da estratégia passiva, $H_{o}:\left(P_{1}+P_{2}\right) \leq 1$; amostra dividida pela magnitude de $\left|R_{M t}-R_{f t}\right| ; 243$ fundos; entre setembro de 1998 e agosto de 2003

\begin{tabular}{|l|c|c|c|c|}
\hline & \multicolumn{2}{|c|}{ PEQUENAS MAGNITUES } & \multicolumn{2}{c|}{ GRANDES MAGNITUDES } \\
\hline & MÉDIA & DESVIO PADRÃO & MÉDIA & DESVIO PADRÃO \\
\hline$\left(\mathrm{P}_{1}+\mathrm{P}_{2}\right)$ & 0,972 & 0,153 & 0,972 & 0,147 \\
\hline & \# DE FUNDOS & $\%$ AMOS TRA & \# DE FUNDOS & $\%$ AM OS TRA \\
\hline $\mathrm{P}_{1}+\mathrm{P}_{2}>1$ a $1 \%$ de significância & 0 & $0,00 \%$ & 12 & $4,94 \%$ \\
$\mathrm{P}_{1}+\mathrm{P}_{2}>1$ a $5 \%$ designificância & 0 & $0,00 \%$ & 36 & $14,81 \%$ \\
$\mathrm{P}_{1}+\mathrm{P}_{2}>1$ a $10 \%$ de significância & 3 & $1,23 \%$ & 51 & $20,99 \%$ \\
\hline $\mathrm{P}_{1}+\mathrm{P}_{2}>1$ & 128 & $52,67 \%$ & 150 & $61,73 \%$ \\
\hline
\end{tabular}

* indica rejeição da hipótese $H_{o}:\left(P_{1}+P_{2}\right) \leq 1$ ao nível de significância de $1 \%$. 
ou em linha com. a hipótese de mercados eficientes em que o padrão ten de a se repetir como remuneração por algum risco sistemático assumido, ou como uma anomalia passível de arbitragem até o seu desaparecimento.

Finalmente, a evidência nacional do sucesso da especulação ativa nas grandes variações não é suficientemente forte para justificar a alocação quase absoluta dos recursos em fundos ativos. A estagnação dos fundos passivos em percentuais relativamente baixos permanece inexplicada, representando tema potencial para pesquisas futuras. Do lado da demanda, cabe uma anál ise micro do grau de conhecimento do investidor sobre a diversidade de opções e seus padrões de retorno, e de suas motivações subjetivas para a escolha. Do lado da oferta, além do respectivo estudo micro abordando questões comerciais, cabem extensões que estudem o desempenho dos fundos passivos, principalmente no que diz respeito à sua efetiva capacidade de acompanhar seu referencial.

\section{NOTA DE AGRADECIMENTO}

Agradecemos à Eurilton Araújo Jr. (Ibmec São Paulo), Antônio Duarte Jr. (Faculdades Ibmec/RJ) e Gyorgy Varga (FCE Modelagem Financeira A plicada) pelos comentários. Agradecemos também à FCE por gentilmente ceder os dados para este trabalho.

\section{REFERÊNCIAS}

BRITO, N. Avaliação do desempenho e market timing: o índice de habilidade. Revista Brasileira de Finanças, v. 1, p. 1-17, 2003.

BRITO, N.; BONA, A.; TARCIRO, A. Estimating Risk and Return Combinations for $\mathrm{N}$ ew Actively Managed Funds. (Texto para discussão). Rio de Janeiro: Coppead/UFRJ, 2003.

FAMA, E. Efficient capital markets: a review of theory and empirical work. Journal of Finance, v. 25, n. 2, p. 383-417, 1970.

FRANZ, P.; FIGUEIREDO, A. Avaliação da capacidade de market timing dos administradores de fundos mútuos de ações no Brasil. Revista de Economia e Administração, v. 2, p. 33-46, 2003.

HENRIKSSON, R. Market timing and mutual fund performance: an empirical investigation. Journal of Business, v. 57, n. 1, p. 73-96, 1984.

HENRIKSSON, R.; MERTON, R. On market timing and investment performance. II. Statistical procedures for evaluating forecasting skills. Journal of Business, v. 54, n. 4, p. 513-533, 1981.

JENSEN, M. The performance of mutual funds in the period 1945-1964. Journal of Finance, v. 23, n. 2, p. 389-416, 1968.

MERTON, R. On market timing and investment performance. I. An equilibrium theory of value for market forecasts. Journal of Business, v. $54, n$. 3, p. 363-406, 1981.

TREYNOR, J.; MAZUY, F. Can mutual funds outguess the market? Harvard Business Review, v. 44, n. 4, p. 131-36, 1966.

\section{Artigo recebido em 24.05.2005. A provado em 13.12.2006.}

\section{Liliana de Magalhães C astro Leusin}

Mestre em Economia Empresarial e Finanças pela Escola de Pós-Graduação em Economia,

Fundação Getulio Vargas

Interesses de pesquisa na área de apreçamento de ativos

E-mail: Ileusin@plenusgestao.com.br

Endereço: Rua Quatá, 300, São Paulo - SP, 04546-042

\section{Ricardo Dias de Oliveira Brito}

Doutor em Economia pela Escola de Pós-Graduação em Economia, Fundação Getulio Vargas

Interesses de pesquisa nas áreas de apreçamento de ativos, custo de capital, estrutura de capital

e política de remuneração

E-mail: RicardoDoB@isp.edu.br

Endereço: Rua Quatá, 300, São Paulo - SP, 04546-042 